\title{
Shorted Annular Slot Antenna (ASA) Matched at Three Different Frequencies
}

\author{
Symeon Nikolaou ${ }^{*}(1)$, George E. Ponchak ${ }^{(2)}$, John Papapolymerou ${ }^{(1)}$ and Manos \\ M. Tentzeris ${ }^{(1)}$. \\ (1) School of ECE, Georgia Institute of Technology, Atlanta, GA 30332-0250 USA. \\ (2) NASA Glenn Research Center, Cleveland, OH 44135 USA. \\ simos@ece.gatech.edu
}

\section{Introduction}

The multitude of different standards in cell phones and other personal mobile devices require multi-band antennas. The use of the same antenna for a number of different purposes, preferably in different frequencies is highly desirable. The shorted Annular Slot Antenna (ASA) proposed in this paper operates in three different frequencies with the central frequency at $5.8 \mathrm{GHz}$. It is fabricated on cheap material and is a compact design suitable for integrating in mobile devices. The annular slot antenna on dielectric material has been described explicitly in [1]. The effect of one shorted point along the circumference has been explored in [2] and the effect of capacitive loading is investigated in [3]. A number of papers use different feeding techniques in order to achieve broader bandwidth [4], or to demonstrate multi-band operation, [5] and [6]. In these designs all resonating frequencies are excited simultaneously. In the proposed design only one frequency resonates at a time which is desirable when there are power issues.

\section{Antenna Design and Fabrication}

The Annular Slot Antenna (ASA) design is presented in fig.1. The shorted slot is printed on the front side, and the feeding line with the matching stubs, are printed on the back side of the board, using standard photolithography. The antenna is fabricated on a $635 \mu \mathrm{m}$ thick, low loss $(\tan \delta=0.0025)$ Rogers RO3006 with $\varepsilon_{\mathrm{r}}=6.15$ and a copper thickness of $18 \mu \mathrm{m}$. The antenna is fed by a $50 \Omega$ microstrip line with width of $0.92 \mathrm{~mm}$ and the matching stubs are also $0.92 \mathrm{~mm}$ wide. The slot has an outer radius $\mathrm{R}=10 \mathrm{~mm}$ and inner radius $8 \mathrm{~mm}$ resulting a slot width of $2 \mathrm{~mm}$. The feeding line is $25 \mathrm{~mm}$ long and the end of the line is at the slot center. Consequently D1 $=15 \mathrm{~mm}$. The stub (1) with $\mathrm{L} 2=4.21$ at $\mathrm{D} 3=7.27 \mathrm{~mm}$ matches the radiating element at the center frequency of $5.8 \mathrm{GHz}$. The simulated radiation efficiency was $92 \%$ at this frequency. With the use of an additional matching stub (2) a different frequency can be matched. When the second stub has length $\mathrm{L} 1=4.98 \mathrm{~mm}$ and $\mathrm{D} 2=5.49 \mathrm{~mm}$, combined with stub (1), the antenna resonates at $5.2 \mathrm{GHz}$. If alternatively $\mathrm{L} 1=3.22 \mathrm{~mm}$ and $\mathrm{D} 2=4.1 \mathrm{~mm}$, combined with stub (1), the slot antenna resonates at $6.4 \mathrm{GHz}$. Since the radiating element radiates with high efficiency in a wide band [4] the problem is the matching in order to avoid reflections before the guided signal actually gets to the slot. Since each matching network is optimized at a certain frequency the whole structure radiates with high efficiency. Simulations showed radiation efficiency higher than $82 \%$ in all three 
different frequencies. The outer part of the slot is used as the ground plane for the microstrip line on the back side. The ground plane occupies most of the required space. The whole board used is $50 \mathrm{~mm} \times 50 \mathrm{~mm}$. $(\mathrm{S}=50 \mathrm{~mm})$ while the slot and the feeding line with the stubs can be confined in a rectangle area $20 \mathrm{~mm}$ x $35 \mathrm{~mm}$ big.

\section{Discussion of Measurements and Simulated Results}

For the return loss and radiation pattern measurements a $3.5 \mathrm{~mm}$ SMA connector was soldered on the microstip line, and an HP 8530A network analyzer was used. The return loss simulations and measurements are presented in fig. 2. For the 5.8 $\mathrm{GHz}$ frequency, measurement matches the simulation in great detail, both in frequency position and in the resonance depth. Return loss lower than $-20 \mathrm{db}$ is measured which implies $99 \%$ radiation. For the lower frequency at $5.2 \mathrm{GHz}$ there is a small shift of the resonance towards lower frequencies. The simulated resonance frequency was $5.2 \mathrm{GHz}$ and the measured was $5.1 \mathrm{GHz}$. A similar small shift is noticed for the higher frequency as well. The simulated resonance frequency was $6.4 \mathrm{GHz}$ and the measured resonance was $6.2 \mathrm{GHz}$. Those small shifts are caused from fabrication and measurement inaccuracies. Especially the discontinuity caused from the soldered connector can cause significant shifts in return loss measurements.

The radiation patterns presented in figs. 3-5 lay on a plane perpendicular to the directivity direction which is parallel to $\mathrm{z}$ axis and also perpendicular to both $\mathrm{E}$ and $\mathrm{H}$ planes. $\mathrm{E}_{\varphi}$ component is measured and simulated. The $\mathrm{E}_{\theta}$ component on that plane (cross polarization) is much smaller (more than $20 \mathrm{~dB}$ ). The $\mathrm{x}$ axis corresponds to $\varphi=0^{\circ}$ and $y$ axis corresponds to $\varphi=90^{\circ}$. The axes orientation with respect to the antenna orientation, appear in fig. 1. Fig. 3 shows the simulated radiation pattern when no short is placed along the circumference of the slot. A null is observed in the feeding line direction. When a short is placed along the circumference, it causes a shift in the radiation pattern as seen in fig.4. Specifically, for the central frequency, the null appears at 180 degree from the location of the short. For the other two frequencies there is a shift in the null position from 180 degree. For the $6.4 \mathrm{GHz}$ frequency, the wavelength is smaller compared to the wavelength for the central frequency and that causes the null to shift towards the short. For the $5.2 \mathrm{GHz}$ frequency, the null direction shifts farther from the short because the wavelength is larger compared to the $5.8 \mathrm{GHz}$ wavelength. Thus, although there is a similar E field distribution along the circumference for all three frequencies, with a minimum at the short, the different wavelength of those frequencies results in a shift in the null position observed in fig. 4. The $\mathrm{E}_{\varphi}$ field is measured for the $5.8 \mathrm{GHz}$ frequency. The simulation and measurement results at $5.8 \mathrm{GHz}$ are presented on the same diagram for comparison in fig. 5. Good agreement is observed. The measurement verifies the simulation in both the direction and the depth of the null. The smaller dip on the pattern, observed close to $115^{\circ}$, is within measurement tolerances. 


\section{Conclusion}

A compact shorted annular slot antenna was presented. The proposed antenna design can be easily fabricated on a low-cost Duroid material and can be easily packaged and integrated with other components. The compact size and the operation frequency make it suitable for personal mobile devices. The simulations agree fairly well with the measurements. All the matching stubs can be printed on the same board and with the selective use of them, a frequency reconfigurable antenna can be designed. The placement of a short along the slot circumference rotates the radiation pattern on the x-y plane. With the appropriate placement of shorts a reconfigurable radiation pattern can also be achieved.

\section{References:}

[1] Tong, C.E.and Blundell, R., “An annular slot antenna on a dielectric half-space”, IEEE Trans. Antennas and Propagation vol 2 , no.7, pp.967 - 974, July 1994.

[2] Morishita, H., Hirasawa, K, Fujimoto, K., " Analysis of a cavity-backed annular slot antenna with one point shorted" Antennas and Propagation, IEEE Trans. Antennas and Propagation, vol.39, no.10, pp.1472 - 1478, October 1991.

[3] Cheng-Shong Hong," Small annular slot antenna with capacitor loading" Electronics Letters, vol.36, no.2, pp.110 - 111, January 2000.

[4] Minard, P., Louzir, A., "A new wide frequency band feeding technique of annular slot antenna", Trans. 2002 IEEE Antennas and Propagation Society International Symposium, Volume: 1 , pp.406 - 409, June 2002, San Antonio, TX.

[5] Hooman Tehrani and Kai Chang, "A multi-frequency microstrip-fed annular slot antenna" Trans. 2000 IEEE Antennas and Propagation Society International Symposium, Volume: 2, pp.632 - 635, July 2000, Salt Lake City, UT.

[6] Le Bolter, F., Louzir, A., " Multi-band annular slot antenna for WLAN applications" Antennas and Propagation, 2001. Eleventh International Conference on (IEE Conf. Publ. No. 480), Volume: 2 , 17-20 April 2001. Pages:529 - 532 vol.2 


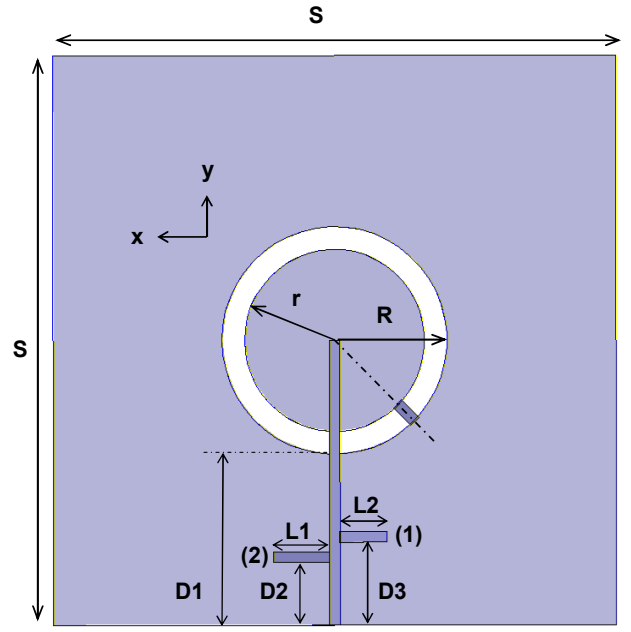

Fig. 1. ASA design. The feeding line and the stubs are in the back side of the board.

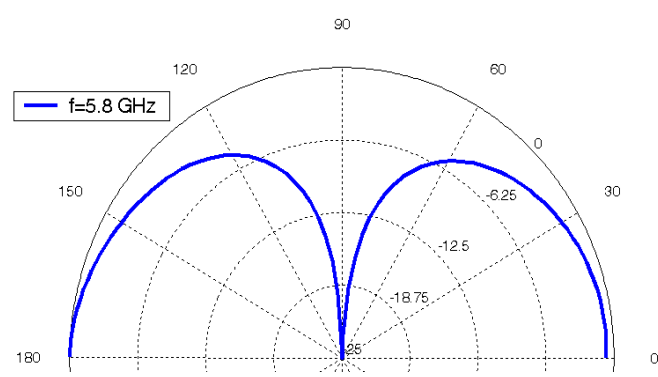

Fig. 3. $\mathrm{E}_{\varphi}$ radiation pattern at $5.8 \mathrm{GHz}$ for slot without short.

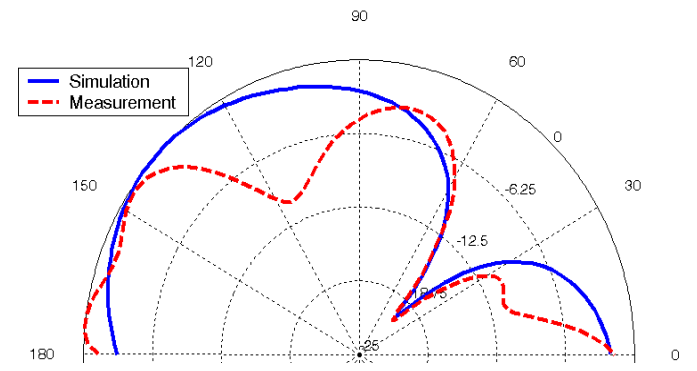

Fig. 5. $\mathrm{E}_{\varphi}$ radiation pattern at $5.8 \mathrm{GHz}$, with the short along the slot circumference.

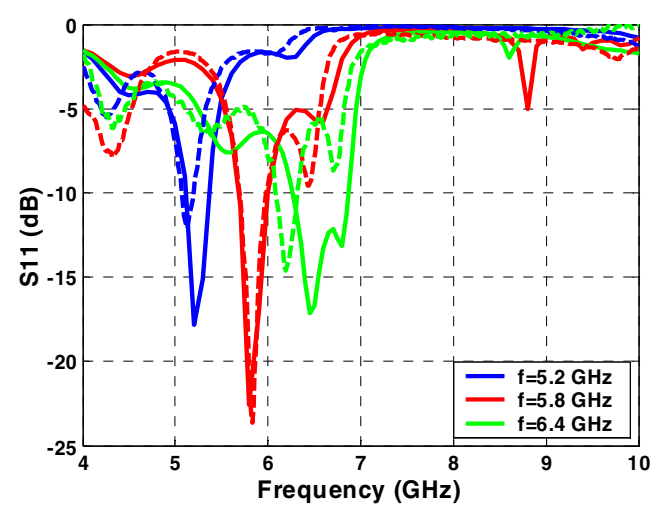

Fig. 2. Return loss. Simulation is in solid line while measurement is in dashed line.

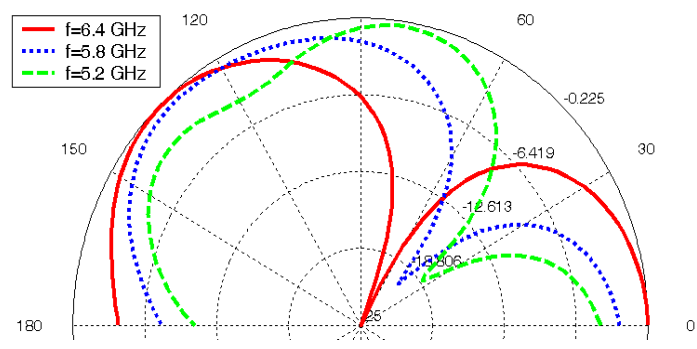

Fig. 4. $\mathrm{E}_{\varphi}$ radiation pattern at three different frequencies. 